Vol 10, Issue 10, 2017

\title{
STARZ-DRP: A TOOL FOR PHARMACY TRIAGE SERVICES
}

\author{
NAZRI NORDIN ${ }^{1 *}$, AZMI SARRIFF ${ }^{1}$, MOHAMED AZMI HASSALI $^{3}$
}

\begin{abstract}
${ }^{1}$ School of Pharmaceutical Sciences, Universiti Sains Malaysia, 11800 Minden, Pulau Pinang; ${ }^{2}$ Department of Clinical Pharmacy, School of Pharmaceutical Sciences, Universiti Sains Malaysia, 11800 Minden, Pulau Pinang, ${ }^{3}$ Department of Social and Administrative Pharmacy, School of Pharmaceutical Sciences, Universiti Sains Malaysia, 11800 Minden, Pulau Pinang. Email: nazri@i-raey.com
\end{abstract}

Received: 12 May 2017, Received and Accepted: 17 June 2017

\section{ABSTRACT}

Objective: The aims of this study are to demonstrate a feasibility study, using an approach, known as STARZ-DRP to counsel people about health complaints and identify drug-related problem (DRPs).

Methods: This study involved community pharmacists (CPs) in the state of Penang, Malaysia, randomly selected as study and control groups, recruiting patients to involve in this study according to the inclusion and exclusion criteria, excepting for control group which were conducted by trained interviewers. The patients had to involve in two-phase study, baseline and post-study. The study pharmacists had been trained to follow the framework known as STARZ-DRP.

Results: Nine CPs agreed to involve in this study, were randomly selected as study ( $\mathrm{n}=5$ ) and control (n=4), recruiting 617 and 636 patients (study, control) accordingly. More male (study $=52.7 \%$; control $=58.2 \%$ ) were recruited. Cough (study $=23.0 \%)$ and nasal problem $($ control $=29.9 \%)$ were indicated as the highest chief complaint by the patients. $81.8 \%$ and $37.1 \%$ of study and control patients should be referred to general practitioners. Significant differences were observed when comparing the mean \pm standard deviation of DRPs between the groups and baseline versus post-study.

Conclusion: STARZ-DRP is promoted as a tool to make triaging decision with evidence base at community pharmacy settings.

Keywords: Community pharmacist, Triaging, Drug-related problem, Self-care practice, Referral.

(C) 2017 The Authors. Published by Innovare Academic Sciences Pvt Ltd. This is an open access article under the CC BY license (http://creativecommons. org/licenses/by/4. 0/) DOI: http://dx.doi.org/10.22159/ajpcr.2017.v10i10.19857

\section{INTRODUCTION}

Pharmaceutical industries are manufacturing wide range of nonprescription, supplementary, and over-the-counter medication, available in the pharmacy without prescription [1]. The products are safe, effective, and cheaper [2]. As a result people start to make own selfdiagnosis, treating themselves with the easily accessible medications in the pharmacy [3]. Nevertheless, this kind of practice emerges some issues such as: Has the disease been cured?; Did I get relief from the symptoms?; Have I got the disease under control?; Was I able to prevent the disease or the symptoms of the disease?; Have I been able to normalize a physiologic parameter? These issues arise since the general public does not use sophisticated clinical, chemical, or mathematical criteria for deciding its drug-related outcomes. Therefore, this practice requires for the community pharmacists (CPs) to take the extended role to counsel people about appropriate self-care practice [4-6]. However, lack of study about this role in Malaysia is catching our attention to conduct this study. This is the first ever study conducted in Malaysia to investigate the potential of CPs to assist their patients to make the right choice of their self-care treatment, using a structured and systematic framework known as STARZ-DRP. The aims of this study are to conduct a feasibility study, using STARZ-DRP Form as a structured and systematic approach to counsel patients about appropriate self-care practice, identifying major illnesses that require for immediate general practitioners' (GPs) attention, and to identify, prevent, and resolve drugrelated problem (DRPs). Rationale of this study is that it can help CPs to identify a potential framework to follow when CPs decide to extend their roles as a self-care advisor at their community pharmacy settings.

\section{METHODS}

Demonstration of the health-care trial project

Seventy-seven pharmacies located in the mainland of the Penang state were invited to attend the demonstration project. The pharmacies were randomly assigned to either a study group or a control group. The study group underwent a training session before the start of the study, in which the participants were presented with STARZ-DRP approach for minor illnesses consultation. STARZ-DRP is developed based on pharmaceutical care concept (Assess, Develop Care Plan, and Establish follow-up to review) [7]. This framework STARZ-DRP stands for a specific definition as depicted in Table 1 and it is presented in a form as depicted in Fig. 1 (for the purpose of documenting and analyzing vital information, especially when the patient comes back for followup session). Each letter represents a sequential step in the decisionmaking process. Presentation of case studies using this form during the training should enable the study pharmacists to translate the acquired knowledge into practice. This approach had been validated by 11 GPs and $17 \mathrm{CPs}$. The pharmacies of the control group did not receive any training and they were continuing with their usual practice (Fig. 2).

Eligible study participants were identified from the patients who visited the pharmacies and either presenting with a specific illness and requested for help or asked by name for medication for a specific illness. For the purpose of the demonstration project, the selected illnesses were a headache, dysmenorrhea, back pain, constipation, dyspepsia, nasal symptoms, sore throat, cough, and high temperature. These illnesses were selected as it had high presentation rate in practice and were considered as appropriate for self-medication with non-prescription medications. Willing patients were enrolled into the study. All patients were informed about the overall study objectives.

The inclusion criteria for the participants were:

1. Age 18 years and above

2. Presenting with the selected minor illnesses

3. Required a product for the treatment of the selected minor illnesses

4. Well-oriented to people, time, and place

5. Staying within $10 \mathrm{~km}$ from the pharmacies

6. Agreement with adhering to the study protocol

7. Signing the informed consent form. 
Table 1: Definition of letters in STARZ\#,*

\begin{tabular}{ll}
\hline Letter & Description \\
\hline $\mathrm{S}$ & Symptom presentation refers to subjective evidence of health problem perceived by the patient \\
$\mathrm{T}$ & Time of onset and duration of the present symptoms \\
$\mathrm{A}$ & Associated symptoms refer to patient symptoms explored and determined by the pharmacist during the interview. It does not refer to \\
& the symptoms presented earlier by the patient. This is done using the pictorial documentation form as depicted in Fig. 1. To aid and ease \\
& the pharmacist during the interview, the human body is arbitrarily divided into four regions: (i) Front: the part of the body facing the \\
& pharmacist (asking for symptoms such as bloating, heartburn, nausea, vomiting, breathlessness, etc.), (ii) Back: (asking for symptoms \\
& such as lower and upper back pain, shoulder pain, and neck pain), (iii) Upper (head) (asking for symptoms such as headache, dizziness, \\
& problems with sleep, etc.,), (iv) Lower (asking for symptoms such a numbness in both legs and hands, constipation, and swollen feet). \\
& $\begin{array}{l}\text { Perhaps, the method is likened to a filtering or screening process to rule out the presence of severe symptoms } \\
\text { R }\end{array}$ \\
& Recurrence problem refers to the symptoms have been treated before, specifically when the symptoms recur and persist despite the \\
treatment prescribed & \\
Z & $\begin{array}{l}\text { Zoom into the patient's medication experience refers to information collected by the pharmacist related to any medical problems (e.g., } \\
\text { hypertension, diabetes, hyperthyroid, etc.), medication utilization (e.g., use of prescription and non-prescription drugs, and herbal } \\
\text { supplements), immunization history, allergies, drug sensitivities, drug side effects, adverse reactions, and the consumption of alcohol, }\end{array}$ \\
& caffeine, and tobacco
\end{tabular}

\#This is not a diagnostic tool, rather it is a format with the purpose of organizing a community pharmacist's knowledge in a manner that allows him/her to begin identifying the actual and potential drug-related problems and subsequently referring triage patients to the appropriate health-care professionals, ${ }^{*}$ The patient's vita signs will be measured when necessary. At times, the patient's blood pressure, pulse rate, and body temperature are measured to aid the pharmacist in assessing the appropriateness of symptoms for self-medication

The exclusion criteria for the participants were:

1. Not fulfilling the study protocol

2. Refusing to sign the informed consent form

3. Showing functional (comprehension, reading, or writing) and/or sensory problems (hearing and/or vision)

The required sample size for the study was calculated on the basis of the population prevalence of minor illnesses in the community pharmacy. With a $95 \%$ confidence level, $60 \%$ of population prevalence of minor illnesses and $5 \%$ precision gave a total of 369 . Adjustments were made for upward to consider a potential dropout rate of $30 \%$ and 480 participants should be enrolled in each group.

This study was commencing from March to May 2009. In both groups, pharmacists exclusively did all self-care consultations. The pharmacy staff did not participate actively in the study. For the control group, exit surveys were done by a trained interviewer to collect data pertaining to the encounter with the pharmacist. All patients were followed for one week and outcomes data were collected by means of standardized questionnaires.

The responses obtained from the study were analyzed using the statistical package SPSS [8]. Descriptive statistic was utilized to present the frequency and mean of the data collected. Comparisons made between the data for both groups using appropriate statistical tests.

Since the study recruited participants with minor illnesses, the risks to the safety of the patients in the trial were low. Furthermore, minor illnesses do not require urgent hospital admission. In fact, a simple non-prescription or over-the-counter medicine is often appropriate to alleviate the symptom presentation. This study commenced after receiving ethical approval from USM-Lam Wah Ee Hospital Joint Committee for Clinical Study Ethics, dated October 17, 2008.

\section{RESULTS}

A total of nine (9) CPs had given their consent to involve in the study, randomly performing as study CPs $(n=5)$ and control CPs $(n=4)$. A total of 617 and 636 study and control patients were recruited, respectively. A significant difference was found between both groups, demonstrating more elderly group in the study group (Table 2). Malay groups were engaged more in both group (study, $n=484,78.4 \%$; control, $n=520$, $81.8 \%$ ). Both groups were recruiting more male (study, $n=325,52.7 \%$; control, $n=370,58.2 \%$ ). Most of the patients were employee (study, $\mathrm{n}=234,37.9 \%$; control, $\mathrm{n}=317,49.8 \%$ ).
Most of the study patients indicated cough ( $n=142,23.0 \%)$ as their main health problem, whereas the control patients indicated about nasal problem ( $\mathrm{n}=190,29.9 \%)$ (Table 2$)$. Both groups indicated period pain (study, $n=3,0.5 \%$; control, $n=3,0.5 \%$ ) as the least reported health problem. In the study and control group, $81.8 \%$ and $37.1 \%$ of patients should be referred to other physicians for further medical examination, respectively. Most of the control patients (62.9\%) were suitable for selfcare treatment, comparing with the study patients (17.5\%) (Table 2).

A significant difference was noted when comparing both groups regarding duration of health complaint and associated symptoms as depicted in Table 2 . The study group presented with longer duration (Mean \pm standard deviation [SD] of 5.06 \pm 3.37 ) and more numbers of associated symptoms were indicated (Mean \pm SD of $5.47 \pm 3.20$ ) than the control group.

Another significant difference was also noted in both groups when comparing inter- and intra-baseline and post-study DRPs (Table 3). In the study group, more DRPs were significantly $(\mathrm{p}<0.01)$ identified in the baseline study (Mean \pm SD of $1.97 \pm 0.85$ ), comparing with poststudy (Mean \pm SD of $1.37 \pm 0.79$ ). In the control group, more DRPs were significantly $(\mathrm{p}<0.01)$ identified in the post-study (Mean \pm SD of $2.01 \pm 1.40$ ), comparing with the baseline (Mean \pm SD of $1.37 \pm 0.79$ ). Noteworthy differences were observed $(\mathrm{p}<0.01)$ when comparing the study and control group during baseline and post-study.

\section{DISCUSSION}

This study is demonstrating the feasibility of STARZ-DRP Form to document patient medical and medication profile and help CPs to analyze candidates that are suitable for self-care treatment and identify DRPs that need to be resolved. The first section in STARZ-DRP Form is developed to help CPs to identify any serious signs and symptoms that require for urgent medical attention. This part in STARZ-DRP Form is encouraging CPs to take more responsibilities, counseling their patients about their current health status, collaborating with patients to develop drug therapy plan, and recommending non-prescription, supplementary, and/or over-the-counter medication appropriately $(17.5 \%)$. In specific condition, the patients have been given some advices on their diets or else $(0.6 \%)$ or others were referred to GPs for further medical examination (81.8\%). These results demonstrate the potential among trained CPs to make a triaging decision, helping the patients to make a quick decision about their condition. A lot of previous studies have revealed self-care practice might have the potential to hide more major health problem if the patients do not seek an appropriate advices [9-12]. In developing countries such as Sudan, the antibiotics 


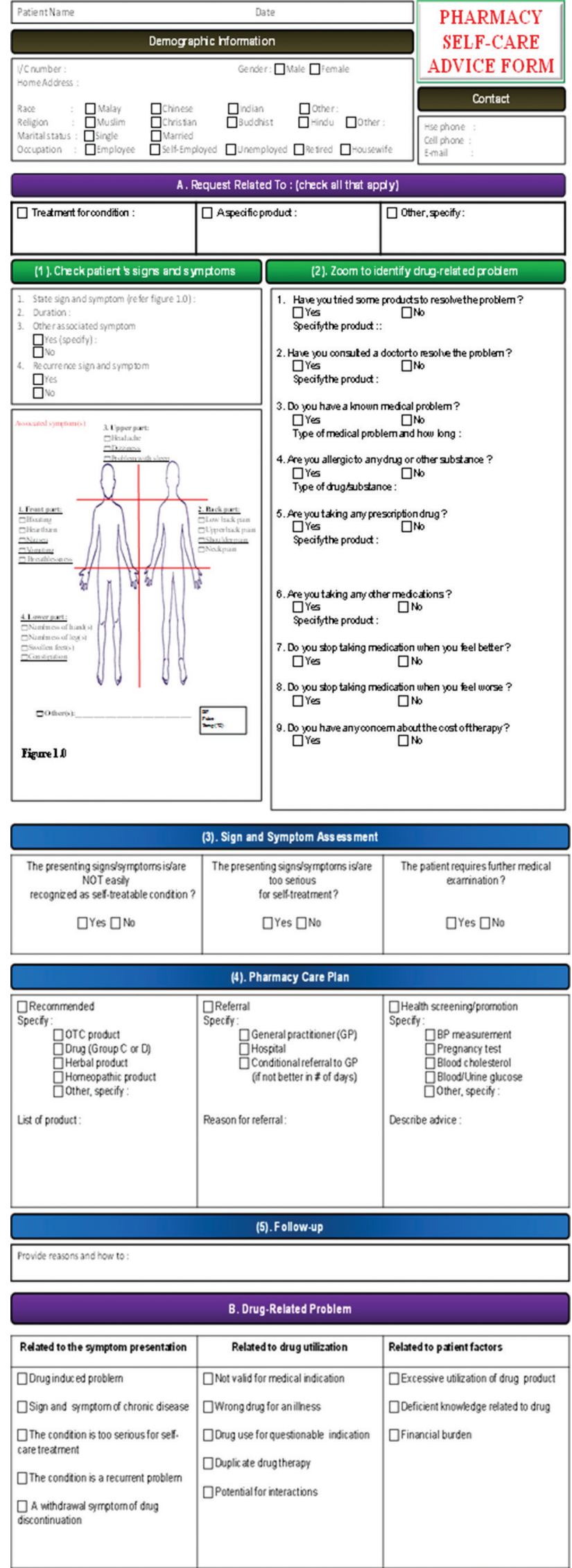

Fig. 1: Pharmacy self-care advice form

and antimalarials are easily accessible in the market, contributing to the inappropriate use among the people [10]. Supposedly, pharmacists must educate the patients to seek advices from GPs if there is a need for the medications. Actually, this is the responsibility of pharmacists, acting as a "gatekeeper" to screen out major illnesses out of minor illnesses, referring when necessary [13]. However, the role requires a structured and systematic approach to follow as a guideline to perform this duty. WWHAM, ASMETHOD, SIT DOWN SIR, ENCORE, CHAPS-FRAPS, and QuEST/SCHOLAR are among the structured and systematic approach, promoted to be used to screen out patients for major illnesses [14-19]. Nevertheless, there are some limitations about these approaches that have been reviewed elsewhere [20]. For example, these approaches are not mainly developed to identify, prevent, and resolve DRPs, as part of its activities. Its performance is to respond to customers' chief complaint, deciding if the health problem needs for urgent medical attention, excluding the roles of CPs to investigate if the problems are caused by some medications. However, our study is demonstrating the feasibility of STARZ-DRP as a systematic and structured framework, using to screen out for major illnesses that need for immediate medical attention and identifying DRPs, adding more value to the previous frameworks. Comparing with other frameworks [14-19], STARZ-DRP is presented in an organized form, using to document patient medical profile, helping CPs to analyze the information, and establish drug therapy plan including referral activities [20]. In this form, CPs are required to establish a follow-up schedule to review the effectiveness of the drug therapy plan, changing to new medications or referring to GPs when necessary, as demonstrated in this study. However, there are no such documenting activities when CPs use other frameworks [14-19]. In addition, other frameworks do not require CPs to establish a follow-up schedule with patients to review the decision made [14-19]. Therefore, the authors are promoting STARZ-DRP Form to be used for pharmacy triage services.

Assessing associated symptoms as demonstrated in STARZ-DRP Form are crossing over the age-old function of CPs, dispensing, and compounding ingredients [21-23]. Despite Doctor of Pharmacy (PharmD) course, other undergraduate program is rarely teaching pharmacy students to conduct a physical examination or ask relevant symptoms [22-24], leaving CPs with low self-confidence [25]. Nevertheless, in this study, the trained CPs are driven to perform their duty, recruiting 617 participants themselves into the study, collecting complete medical profile, analyzing the data, and establishing an exclusive therapy plan for individual patients. This scenario is revealing that ongoing training can help CPs to enhance their self-confidence and personal perception of their competence, even in the developed countries such as United Kingdom [26] and Australia [27]. In the United Kingdom, for example, the Royal Pharmaceutical Society is organizing an accredited program for CPs to be a supplementary prescribing, ending with graduates who are motivated to perform the duty, side-by-side with other GPs [26]. The study is revealing that CPs are potential to perform the extended roles on condition that they are ready to enhance their knowledge and skills.

Holland in his book has outlined a list of symptoms which are too fundamental for self-care treatment, requiring CPs to assess it among their patients before suggesting medications [28]. This list can serve as a guideline when CPs are conducting the assessment, using the STARZ-DRP Form. Interestingly, the symptoms might be presenting actual or potential DRPs that need to be resolved, instead of suggesting medications to alleviate the symptoms. In this study, the trained CPs are able to demonstrate about this scenario, rating patient medication profile, and identifying problems induced by medications, and the problems have been resolved as depicted in Table 3. This study is revealing that CPs must be cautious with patients who ask for selfcare medications as their symptoms might hide actual or potential problems. Other studies, for example, reveal non-steroidal antiinflammatory drugs (NSAIDs) induced upper and lower gastrointestinal symptoms [29-32] and cardiovascular risk [32] and the patients might need to stop the medications before it becomes worse. Certainly, most of NSAIDs are reachable in the pharmacy, requiring CPs to take the role to counsel their patients about the potential side effects [33,34]. This role is a shifting focus among CPs to be more on patient-oriented rather 


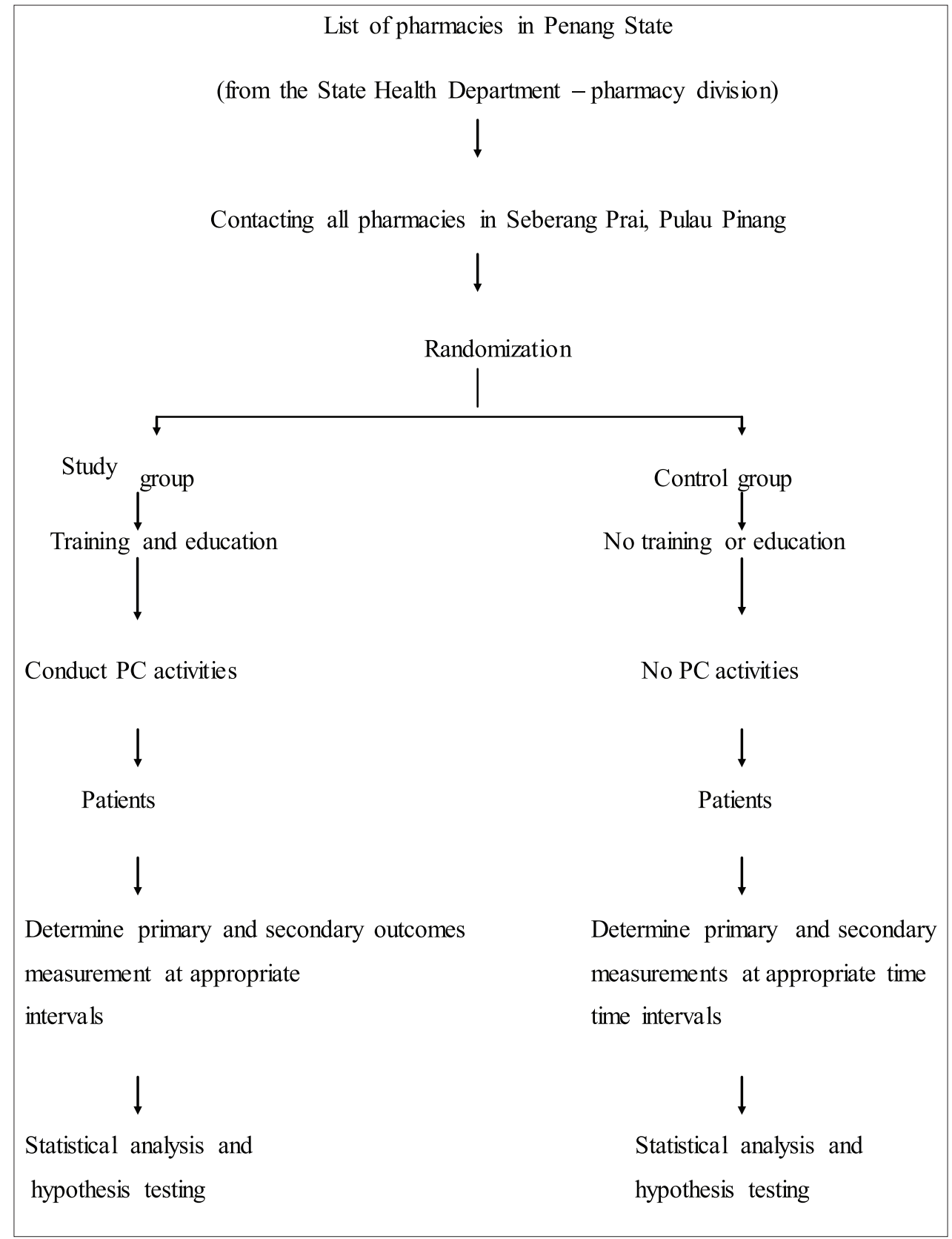

Fig. 2: Flow chart of the randomized health-care trial. Notes: PC - pharmaceutical care; Trained interviewers documented control CPs activities; Study CPs documented own activities

than product-oriented for the safety of the patients. Patient-oriented requires CPs to make sure each medication consumed by patients should be safe and effective $[11,13]$. Therefore, in our study, a significant difference is observed when comparing DRPs in baseline (Mean \pm SD of $1.97 \pm 0.85$ ) and post-study (Mean \pm SD of $1.37 \pm 0.79$ ), revealing the trained CPs have established safe and effective drug therapy plan for individual patients and eliminating DRPs. This extended role is also observed in other previous studies, eliminating DRPs after intervention by CPs, especially DRPs related to inappropriate medication use among self-medicate customers. Cooper in his review reveals a list of overthe-counter medication abuse among the customers, requiring CPs to aware of their customers' request for [35]. Among the medication abuse is non-opiate cough medication which is easily accessible at the community pharmacy. Assessing for more information about the customers' medical and medication profile can help CPs to promote appropriate medication use, like our trained CPs do. Another example, a relevant study which conducted in Germany reveals a potential of CPs to document DRPs among customers who decide to self-medicate, utilizing a standard documentation form [36]. Among identified DRPs were self-medication was inappropriate, requested product was inappropriate, wrong dosage, contraindication, wrong use of drug, duplication of drug therapy, drug-drug interaction, and adverse drug reaction, exactly like the outcome of our study. CPs had taken urgent action to prevent and resolve the problem, acting like our trained CPs. Other relevant study conducted in Danish reveals a potential of CPs to document DRPs among customers who request for over-the-counter medication, demonstrating a list of DRPs such as inappropriate choice of self-medication, adverse medication events, duplicate medication therapy, interaction between medications, medication is taken too long or too short, contraindication and wrong use of medication [37]. The outcome of the study is the same as our study when the spectrum of those DRPs is also identified among our control patients who ask for self-medication. It shows that CPs must aware of potential among selfmedicate patients to suffer of unwanted drug related issues, requiring CPs to take responsibilities to advise their customers about appropriate self-care treatment. For example, when analyzing the control group, a 
Table 2: Sociodemographic characteristics of patients

\begin{tabular}{|c|c|c|c|}
\hline \multirow[t]{2}{*}{ Characteristics } & \multicolumn{2}{|l|}{ Frequency (\%) } & \multirow[t]{2}{*}{$\mathbf{p}^{*}$} \\
\hline & Study $(n=617)$ & Control $(n=636)$ & \\
\hline \multicolumn{4}{|l|}{ Age } \\
\hline 18-64 years & $549(89.0)$ & $614(96.5)$ & $<0.001$ \\
\hline$>64$ years & $68(11.0)$ & $22(3.5)$ & \\
\hline Mean \pm SD & $46.20 \pm 15.37$ & $40.82 \pm 13.20$ & \\
\hline Median & 47.00 & 39.00 & \\
\hline \multicolumn{4}{|l|}{ Gender } \\
\hline Female & $292(47.3)$ & $266(41.8)$ & \\
\hline Male & $325(52.7)$ & $370(58.2)$ & \\
\hline \multicolumn{4}{|l|}{ Race } \\
\hline Malay & $484(78.4)$ & $520(81.8)$ & \\
\hline Chinese & $9(1.5)$ & $35(5.5)$ & \\
\hline Indian & $114(18.5)$ & $73(11.5)$ & \\
\hline Others & $10(1.6)$ & $8(1.2)$ & \\
\hline \multicolumn{4}{|l|}{ Religion } \\
\hline Muslim & $494(80.1)$ & $525(82.5)$ & \\
\hline Hindu & $98(15.9)$ & $62(9.7)$ & \\
\hline Buddhist & $9(1.5)$ & $38(6.0)$ & \\
\hline Others & $1(0.2)$ & $3(0.5)$ & \\
\hline \multicolumn{4}{|l|}{ Occupation } \\
\hline Employee & 234 (37.9) & 317 (49.8) & \\
\hline Homemaker & 177 (28.7) & $94(14.8)$ & \\
\hline Retired & $26(4.2)$ & $42(6.6)$ & \\
\hline Self-employed & $124(20.1)$ & $110(17.3)$ & \\
\hline Unemployed & $56(9.1)$ & $73(11.5)$ & \\
\hline \multicolumn{4}{|l|}{ Reason for visiting the pharmacy } \\
\hline Complaining about health issue & $611(99.0)$ & $539(84.7)$ & \\
\hline Request for a specific product & $6(1.0)$ & $97(15.3)$ & \\
\hline \multicolumn{4}{|l|}{ Type of health complaint } \\
\hline Headache & $49(7.9)$ & $46(7.2)$ & \\
\hline Period pain & $3(0.5)$ & $3(0.5)$ & \\
\hline Back pain & $132(21.4)$ & $75(11.8)$ & \\
\hline Cough & $142(23.0)$ & $142(22.3)$ & \\
\hline Nasal problem & $55(8.9)$ & $190(29.9)$ & \\
\hline Abdominal problem & $108(17.5)$ & $10(1.6)$ & \\
\hline Constipation & $18(2.9)$ & $29(4.6)$ & \\
\hline \multicolumn{4}{|l|}{ Consultation time (minute) } \\
\hline Mean \pm SD & $8.35 \pm 3.69$ & $5.87 \pm 1.86$ & \\
\hline Median & 7.00 & 5.00 & \\
\hline \multicolumn{4}{|l|}{ Triage decision } \\
\hline Suitable for self-care treatment & $108(17.5)$ & $400(62.9)$ & \\
\hline Not treated & $4(0.6)$ & 0 & \\
\hline Referral to doctor & $505(81.8)$ & $236(37.1)$ & \\
\hline \multicolumn{4}{|l|}{ Duration (days) } \\
\hline Mean \pm SD & $5.06 \pm 3.37$ & $3.41 \pm 3.30$ & $<0.001$ \\
\hline \multicolumn{4}{|l|}{ Associated symptoms } \\
\hline Mean \pm SD & $5.47 \pm 3.20$ & $2.94 \pm 2.20$ & $<0.001$ \\
\hline \multicolumn{4}{|l|}{ Recurrence problem } \\
\hline Mean \pm SD & $360 \pm 58.3$ & $66 \pm 10.4$ & \\
\hline
\end{tabular}

*Independent sample t-test. Significance was indicated by $\mathrm{p}<0.01$, SD: Standard deviation

significant difference is observed when comparing DRPs in baseline (Mean \pm SD of $1.41 \pm 0.73$ ) and post-study (Mean \pm SD of $2.01 \pm 1.40$ ), revealing DRPs related to deficient knowledge of drug as the most addressed in the post-study. The study is revealing that the untrained CPs might not be aware of improving patients' knowledge about drugs or eliminating DRPs even though this is the actual role of CPs. Potential factors toward this scenario might be lack of interaction with patients, time, knowledge, and skills or patients are in a rush as indicated in other articles [22,26,38,39].

Actually STARZ-DRP Form is developed to encourage CPs to be more responsible for their decision. All decisions including drug therapy plan, referral to other physician, eliminating DRPs, and outcome of the drug therapy plan must be documented for the future review.
Ongoing training can enhance self-confidence and self-competency perception. However, other studies indicate some pharmacists refuse to take responsibilities on their clinical intervention [40-44]. Among their reasons are lack of knowledge and skills [40-44], interaction with patients, [40,44] and experiences [44]. Therefore, the authors have started to teach this first version STARZ-DRP to the pharmacy students, enhancing their knowledge, skills, and experiences, motivating them to interact with patients, and be more responsible with their decision made. The second version STARZ-DRP is an ongoing study in the field, assuming to finish within these couples of months.

This study reveals STARZ-DRP can be used as a universal model for establishing collaboration working relationship between CPs and GPs. According to the study's guidelines, patients who indicate signs and 
Table 3: Baseline and post-study drug-related problems

\begin{tabular}{|c|c|c|}
\hline Type of DRPs & $\begin{array}{l}\text { Frequency, } \\
\text { n (\%) }\end{array}$ & Mean \pm SD \\
\hline \multicolumn{3}{|l|}{ Baseline } \\
\hline \multicolumn{3}{|l|}{ Study $(\mathrm{n}=546)$} \\
\hline Drug-induced problem & $38(7.0)$ & $1.97 \pm 0.85$ \\
\hline Sign and symptom of chronic disease & $161(29.5)$ & \\
\hline Too serious for self-care treatment & $450(82.4)$ & \\
\hline Recurrence problem & 316 (57.9) & \\
\hline Deficient knowledge of drug & $24(4.4)$ & \\
\hline Financial burden & $93(17.0)$ & \\
\hline \multicolumn{3}{|l|}{ Control (n=239) } \\
\hline Drug-induced problem & $3(1.3)$ & $1.41 \pm 0.73$ \\
\hline Sign and symptom of chronic disease & $106(44.4)$ & \\
\hline Too serious for self-care treatment & $146(61.1)$ & \\
\hline Recurrence problem & $68(28.5)$ & \\
\hline Drug with no valid medical indication & $1(0.4)$ & \\
\hline Wrong drug & $1(0.4)$ & \\
\hline Duplicate drug therapy & $3(1.3)$ & \\
\hline Potential drug-drug interaction & $2(0.8)$ & \\
\hline Excessive drug utilization & $4(1.7)$ & \\
\hline Deficient knowledge of drug & $1(0.4)$ & \\
\hline Financial burden & $1(0.4)$ & \\
\hline \multicolumn{3}{|l|}{ Post-study } \\
\hline \multicolumn{3}{|l|}{ Study $(\mathrm{n}=98)$} \\
\hline Sign and symptom of chronic disease & $16(16.3)$ & $1.37 \pm 0.79$ \\
\hline Too serious for self-care treatment & 15 (15.3) & \\
\hline Recurrence problem & $20(20.4)$ & \\
\hline Deficient knowledge of drug & $2(2.0)$ & \\
\hline \multirow{2}{*}{\multicolumn{3}{|c|}{ Control $(n=485)$}} \\
\hline & & \\
\hline Drug-induced problem & $2(0.4)$ & $2.01 \pm 1.40$ \\
\hline Sign and symptom of chronic disease & $15(3.1)$ & \\
\hline Too serious for self-care treatment & $17(3.5)$ & \\
\hline Recurrence problem & $8(1.6)$ & \\
\hline Symptom of drug discontinuation & $2(0.4)$ & \\
\hline Drug with no valid medical indication & $52(10.7)$ & \\
\hline Wrong drug & $40(8.2)$ & \\
\hline Drug with questionable indication & $62(12.8)$ & \\
\hline Duplicate drug therapy & $136(28.0)$ & \\
\hline Potential drug-drug interaction & $30(6.2)$ & \\
\hline Excessive drug utilization & $166(34.2)$ & \\
\hline Deficient knowledge of drug & 441 (90.9) & \\
\hline
\end{tabular}

Trained CPs identified DRPs among study patients; Researchers identified DRPs among control patients. $\mathrm{SD}=$ Standard deviation, CPs: Community pharmacists, DRPs: Drug-related problems

symptoms such as long duration, complicated, and relapse shall be referred to GPs. This standard protocol is allocating space for CPs to perform their roles until the space requires GPs to take over. As a result, this study reveals a clear vision of CPs' and GPs' roles, avoiding CPs to act as GPs, and allocating GPs as primary health-care practitioners. In addition, STARZ-DRP Form allows the trained CPs to communicate with GPs about their referral patients, using common pharmaceutical language, eliminating language barriers between CPs and GPs. Therefore, STARZ-DRP Form is answering to the several barriers indicated in previous studies including lack of standard model for CPs to perform [39,45-47] and lack of collaboration with GPs [26,38,39,48,49].

\section{CONCLUSION}

This study demonstrates the potential of STARZ-DRP Form to be used as a systematic and structured approach to screen outpatient medical profiles for seriousness illnesses that require for immediate medical attention. In addition, the approach is helping CPs to identify, prevent, and resolve DRPs among patients who have intention to self-medicate. This extended role has potential to enhance the image of pharmacy profession, improving the safe use of medication among patients, and increasing effectiveness of medications. Hopefully, STARZ-DRP form can be promoted for CPs, spanning around the world to provide high standard triage services.

\section{ACKNOWLEDGMENT}

We are grateful to the Universiti Sains Malaysia for providing the Research University (RU) Grant to fund this research. Under this RU grant, we are currently conducting a research project entitled "Establishing and implementing the philosophy of pharmaceutical care in the community pharmacy practice - A Malaysian perspective" (Grant number: 1001/PFARMASI/8120234).

\section{REFERENCES}

1. Hussain A, Khanum A. Self medication among university students of Islamabad, Pakistan - A preliminary study. South Med Rev 2008;1(1):14-6.

2. Bolanos BS. Responsible self-medication in latin America. Drug Inf J 2005:39:99-107.

3. Verma RK, Mohan L, Pandey M. Evaluation of self medication among professional student in North India: Proper statutory drug control must be implemented. Asian J Pharm Clin Res 2010;3:60-4.

4. You JH, Wong FY, Chan FW, Wong EL, Yeoh EK. Public perception on the role of community pharmacists in self-medication and self-care in Hong Kong. BMC Clin Pharmacol 2011;11:19.

5. Vohra S. A community pharmacy minor ailment scheme - Effective, rapid, and convenient. Pharm J 2006;276(7406):754-6.

6. Paudyal V, Hansford D, Cunningham S, Stewart D. Pharmacy assisted patient self care of minor ailments: A chronological review of UK health policy documents and key events 1997-2010. Health Policy 2011;101:253-9.

7. Cipolle RJ, Strand LM, Morley PC. Pharmaceutical Care Practice: The Clinician's Guide. $2^{\text {nd }}$ ed. New York: McGraw-Hill; 2004.

8. SPSS. Statistical Package for the Social Sciences. (SPSS 18) for Windows. Version 18. Chicago: SPSS Inc.; 2010.

9. Oshikoya KA, Senbanjo IO, Njokanma OF. Self-medication for infants with colic in Lagos, Nigeria. BMC Pediatr 2009;9:9.

10. Awad A, Eltayeb I, Matowe L, Thalib L. Self-medication with antibiotics and antimalarials in the community of Khartoum State, Sudan. J Pharm Pharm Sci 2005;8:326-31.

11. Covington TR. Non prescription medications and self-care. Non prescription drug therapy: Issues and opportunities. Am J Pharm Educ 2006;70:137.

12. Afolabi AO. Drug use and misuse in an adult Nigerian population. Clin Rev J 2007;68:7-14

13. Smith M, Bates DW, Bodenheimer T, Cleary PD. Why pharmacists belong in the medical home. Health Aff (Millwood) 2010;29:906-13.

14. Blenkinsopp A, Paxton P, Blenkinsopp J. Symptoms in the Pharmacy: A Guide to the Management of Common Illness. $5^{\text {th }}$ ed. Oxford, UK: Blackwell Publishing; 2005. p. 1-13.

15. Edwards C, Stillman P. Minor Illness or Major Disease? The Clinical Pharmacist in the Community. $4^{\text {th }}$ ed. London: Pharmaceutical Press; 2006. p. 1-10.

16. Rutter P. Community Pharmacy: Symptoms, Diagnosis and Treatment. London: Churchill Livingstone, An Imprint of Elsevier Limited; 2004. p. 9-11.

17. Rutter PM, Horsley E, Brown DT. Evaluation of community pharmacists' recommendations to standardized patient scenarios. Ann Pharmacother 2004;38:1080-5.

18. McCallian DJ, Cheigh NH. The pharmacist's role in self-care. J Am Pharm Assoc (Wash) 2002;42 5 Suppl 1:S40-1.

19. Buring SM, Kirby J, Conrad WF. A structured approach for teaching students to counsel self-care patients. Am J Pharm Educ 2007;71:8.

20. Sarriff A, Nordin N, Hassali MA. STARZ-DRP: A step-by-step approach for pharmacy triage services. Mal J Pharm 2011;1(9):311-25.

21. Allen LV. A History of Pharmaceutical Compounding. Vol. 11. No (3). Oklahoma City: Paddock Lab Inc.; 2011. Available from: http://www. perrigo.com/business/pdfs/Sec\%20Artem\%2011.3.pdf.

22. Yamamura S, Yamamoto $\mathrm{N}$, Oide S, Kitazawa S. Current state of community pharmacy in Japan: Practice, research, and future opportunities or challenges. Ann Pharmacother 2006;40:2008-14.

23. Poudel A, Khanal S, Kadir A, Palaian S. Perception of Nepalese community pharmacists towards patient counseling and continuing pharmacy education program: A multicentric study. J Clin Diagn Res 2009;2(3):1408-13.

24. Salim AM, Elgizoli B. Exploring self-perception of community pharmacists of their professional identity, capabilities, and role expansion. J Res Pharm Pract 2016;5:116-20.

25. Malangu N. The future of community pharmacy practice in South 
Africa in the light of the proposed new qualification for pharmacists: Implications and challenges. Glob J Health Sci 2014;6:226-33.

26. Warchal S, Brown D, Tomlin N, Portlock J. Attitudes of successful candidates of supplementary prescribing courses to their training and their extended roles. Pharm J 2006;276:348-52.

27. Berbatis CG, Sunderland VB, Joyce A, Bulsara M, Mills C. Enhanced pharmacy services, barriers and facilitators in Australia's community pharmacies: Australia's National Pharmacy Database Project. Int J Pharm Pract 2007;15(3):185-91.

28. Holland R. When to refer. Pharmaceutical Society of Australia. Sydney: Pharmaceutical Society of Australia; 1993. p. 15

29. Abay SM, Amelo W. Assessment of self-medication practices among medical, pharmacy, and health science students in Gondar University, Ethiopia. J Young Pharm 2010;2:306-10.

30. Lucas R, Lunet N, Carvalho R, Langa J, Muanantatha M, Nkunda LP, et al. Patterns in the use of medicines by University students in Maputo, Mozambique. Cad Saude Publica 2010;23(12):2845-52

31. Sostres C, Gargallo CJ, Lanas A. Nonsteroidal anti-inflammatory drugs and upper and lower gastrointestinal mucosal damage. Arthritis Res Ther 2013;15 Suppl 3:S3.

32. Al-Saeed A. Gastrointestinal and cardiovascular risk of nonsteroidal anti-inflammatory drugs. Oman Med J 2011;26:385-91.

33. Teichert M, Griens F, Buijs E, Wensing M, De Smet PA. Effectiveness of interventions by community pharmacists to reduce risk of gastrointestinal side effects in nonselective nonsteroidal antiinflammatory drug users. Pharmacoepidemiol Drug Saf 2014;23:382-9.

34. Bear MD, Bartlett D, Evans P. Pharmacist counseling and the use of nonsteroidal anti-inflammatory drugs by older adults. Consult Pharm 2017;32:161-8.

35. Cooper RJ. Over-the-counter medicine abuse - A review of the literature. J Subst Use 2013;18:82-107.

36. Hämmerlein A, Griese N, Schulz M. Survey of drug-related problems identified by community pharmacies. Ann Pharmacother 2007;41:1825-32.

37. Frøkjær B, Bolvig T, Griese N, Herborg H, Rossing C. Prevalence of drug-related problems in self-medication in Danish community pharmacies. Innov Pharm 2012;3(4):1-10.

38. Wong FY, Chan FW, You JH, Wong EL, Yeoh EK. Patient selfmanagement and pharmacist-led patient self-management in Hong
Kong: A focus group study from different healthcare professionals' perspectives. BMC Health Serv Res 2011;11:121.

39. Laliberté MC, Perreault S, Damestoy N, Lalonde L. Ideal and actual involvement of community pharmacists in health promotion and prevention: A cross-sectional study in Quebec, Canada. BMC Public Health 2012;12:192.

40. Fang Y, Yang S, Zhou S, Jiang M, Liu J. Community pharmacy practice in China: Past, present and future. Int J Clin Pharm 2013;35:520-8.

41. Austin Z, Gregory PA, Martin JC. Negotiation of interprofessional culture shock: The experiences of pharmacists who become physicians. J Interprof Care 2007;21:83-93.

42. Blake KB, Madhavan SS. Perceived barriers to provision of medication therapy management services (MTMS) and the likelihood of a pharmacist to work in a pharmacy that provides MTMS. Ann Pharmacother 2010;44:424-31.

43. Wheeler A, Crump K, Lee M, Li L, Patel A, Yang R, et al. Collaborative prescribing: A qualitative exploration of a role for pharmacists in mental health. Res Social Adm Pharm 2012;8:179-92.

44. Frankel GE, Austin Z. Responsibility and confidence: Identifying barriers to advanced pharmacy practice. Can Pharm J (Ott) 2013;146:155-61.

45. Egorova SN, Akhmetova T. Pharmaceutical counseling: Between evidence-based medicine and profits. Int J Risk Saf Med 2015;27 Suppl 1:S87-8.

46. Sadek MM, Elnour AA, Al Kalbani NM, Bhagavathula AS, Baraka MA, Aziz AM, et al. Community pharmacy and the extended community pharmacist practice roles: The UAE experiences. Saudi Pharm J 2016;24:563-70.

47. Dol HS, Jadhav SS, Pisal MA, Shaikh SK, Shinde VR. Emerging trends in patient counselling: Current scenario. Int J Pharm Pharm Sci 2015;7(10):65-8

48. Scheerder G, De Coster I, Van Audenhove C. Pharmacists' role in depression care: A survey of attitudes, current practices, and barriers. Psychiatr Serv 2008;59:1155-60.

49. Ali H, Zafar F, Alam S, Mallick N, Hasnain H, Naqvi GR, et al. Integration of community setup in pharmaceutical care: Current challenges, perception, facts and opportunities in Pakistan. Int J Pharm Pharm Sci 2016;8(10):138-43. 